\title{
Corilagin induces apoptosis and inhibits autophagy of HL-60 cells by regulating miR-451/HMGB1 axis
}

\author{
SHU JIA ${ }^{1 *}$, DONGYE HE ${ }^{1 *}$, XIAO LIANG $^{2}$, PANPAN CHENG ${ }^{3}$, JILAN LIU ${ }^{1}$, \\ MINGTAI CHEN ${ }^{1}$, CUILING WANG ${ }^{3}$, HAO ZHANG ${ }^{3}$ and CHUNYANG MENG $^{2}$ \\ ${ }^{1}$ Medical Research Center, Affiliated Hospital of Jining Medical University; Departments of ${ }^{2}$ Spine Surgery and \\ ${ }^{3}$ Hematology, Affiliated Hospital of Jining Medical University, Jining, Shandong 272029, P.R. China
}

Received December 31, 2020; Accepted August 12, 2021

DOI: $10.3892 / \mathrm{mmr} .2021 .12550$

\begin{abstract}
Corilagin is the primary active component of the Euphorbia phyllanthus plant and has significant anti-cancer properties. However, the biological effects and mechanisms of corilagin on acute myeloid leukemia (AML) have not been clarified. The Cell Counting Kit-8 and Carboxyfluorescein Diacetate Succinimidyl Ester assay results showed that corilagin significantly inhibited proliferation of the AML cell line HL-60 in a time- and dose-dependent manner. Western blotting and flow cytometry analysis were performed to determine the levels of apoptosis in HL-60 cells. The protein levels of cleaved caspase-3 and Bak were upregulated, while Bcl-xl was downregulated in cells treated with corilagin. The percentage of early- and late-stage apoptotic cells increased following corilagin treatment in a dose-dependent manner, indicating that the intrinsic mitochondrial apoptosis pathway was activated by corilagin. Simultaneously, western blotting and immunofluorescence results revealed that autophagy was suppressed; this was accompanied by a decrease in light chain 3-II (LC3-II) conversion and autophagosomes. MicroRNA (miRNA/miR) profile analysis showed that corilagin elevated the expression of the tumor suppressor miR-451, while the mRNA and protein levels of high mobility group protein B1 (HMGB1), the target of miR-451, decreased following exposure to corilagin. Knockdown of miR-451 decreased the downregulation of HMGB1 caused by corilagin, indicating negative regulation of
\end{abstract}

Correspondence to: Professor Chunyang Meng, Department of Spine Surgery, Affiliated Hospital of Jining Medical University, 89 Guhuai Road, Jining, Shandong 272029, P.R. China

E-mail: mengchunyang1600@mail.jnmc.edu.cn

Professor Hao Zhang, Department of Hematology, Affiliated Hospital of Jining Medical University, 89 Guhuai Road, Jining, Shandong 272029, P.R. China

E-mail: gx-zhanghao@126.com

${ }^{*}$ Contributed equally

Key words: corilagin, apoptosis, autophagy, miR-451, high mobility group protein B1, acute myeloid leukemia
HMGB1 by miR-451 during corilagin treatment. Furthermore, knockdown of miR-451 also attenuated corilagin-induced proliferation inhibition of HL-60 cells, implying that miR-451 was essential for the proliferation inhibitory effect of corilagin. In conclusion, these results indicated that corilagin induced apoptosis and inhibited autophagy in HL-60 cells by regulating the miR-451/HMGB1 axis, and corilagin may be a novel therapeutic drug for the treatment of AML.

\section{Introduction}

Acute myeloid leukemia (AML) is characterized by blocked myeloid differentiation and abnormal proliferation of immature myeloid cells in the bone marrow and peripheral blood $(1,2)$. The current clinical treatments for AML include induced differentiation therapy, combined chemotherapy, hematopoietic stem cell transplantation and immunotherapy (3-6). Although progress has been made in medicine and chemotherapy, the treatment outcomes of AML are not ideal. Only $35-40 \%$ of young (age $<60$ years) and $5-15 \%$ of elderly patients with AML (age $\geq 60$ years) survive for $>5$ years post-treatment (7). The primary reason for therapeutic failure and poor prognosis is the chemotherapy resistance of leukemia cells $(8,9)$. Therefore, there is an urgent need to find more effective drugs and therapies for treating AML.

Corilagin is the primary active ingredient of the Euphorbia phyllanthus plant and has antiviral, anti-inflammatory, antioxidant and other biological activity (10-12). Corilagin can effectively diminish inflammation in ulcerative colitis and cystic fibrosis bronchial cells. By blocking the activation of nuclear factor $\kappa \mathrm{B}(\mathrm{NF}-\kappa \mathrm{B})$, corilagin decreases expression of interleukin (IL)-1 $\beta$, IL-6, TNF- $\alpha$ and other inflammatory factors $(13,14)$. Previous studies have shown that corilagin has a significant inhibitory effect on glioblastoma, as well as breast and ovarian cancer (15-17). Corilagin inhibits the proliferation of breast cancer cells by inducing apoptosis and autophagy and that of ovarian cancer cells by blocking the typical Smad and atypical ERK/AKT pathways $(16,17)$. Although corilagin possesses anti-inflammatory and antitumor effects, to the best of our knowledge, its function in inhibiting leukemia has rarely been reported.

MicroRNA (miRNAs/miRs) are a class of small, non-coding RNAs with 19-25 nucleotides (nts) that negatively 
regulate target gene expression at the post-transcriptional level. Abnormal expression of miRNAs is associated with a variety of human diseases, including AML (18-20). Modulation of dysregulated miRNAs is used in AML therapy and sensitizes AML cells to chemotherapy $(21,22)$. Overexpression of miR-34a, which is expressed at low levels in AML, promotes apoptosis and inhibits autophagy in AML cells (23). Upregulation of miR-142-3p improves the drug sensitivity of AML cells by inhibiting cell viability and promoting apoptosis (24). Additionally, miR-451 is abnormally downregulated in patients with AML, and miR-451 acts as a tumor suppressor by repressing malignant proliferation and inducing apoptosis $(25,26)$. Upregulation of miR-451 can prevent cardiomyocyte anoxia/reoxygenation $(\mathrm{A} / \mathrm{R})$ injury by suppressing high mobility group protein B1 (HMGB1) (27). However, the association between miR-451 and HMGB1 in AML remains unclear.

HMGB1 is a highly conserved and widely expressed non-histone, chromatin-binding protein, which can bind with specific chromatin DNA and participate in DNA recombination, gene transcription regulation, cell division, and differentiation $(28,29)$. Under metabolic stress, HMGB1 transfers from the nucleus to the cytoplasm and binds Beclin-1 to prevent its aggregation, thus promoting autophagy and inhibiting apoptosis (30). HMGB1 can induce chemotherapy resistance by promoting autophagy in lung and colorectal cancer, as well as other types of tumor $(31,32)$. HMGB1 levels in the serum of patients with AML increase significantly following chemotherapy, and inhibition of HMGB1 can improve the sensitivity of AML cells to chemotherapy drugs $(24,33)$. Therefore, HMGB1 may be an important clinical target for the treatment of leukemia.

In the present study, the effects of corilagin-induced proliferation inhibition were investigated in the AML cell line HL-60. The effect of corilagin on apoptosis and autophagy in AML cells was investigated. The expression levels of miR-451 and HMGB1 were evaluated following corilagin treatment. Finally, the role of miR-451 in corilagin-induced proliferation inhibition on HL-60 cells was assessed. The present study aimed to elucidate the effect and potential mechanism of corilagin on AML cells, which may provide a promising treatment for AML.

\section{Materials and methods}

Cell culture and treatment. The human promyelocytic leukemia cell line HL-60, obtained from the American Type Culture Collection, was maintained in DMEM supplemented with $10 \%$ FBS and $100 \mathrm{U} / \mathrm{ml}$ penicillin/streptomycin at $37^{\circ} \mathrm{C}$ under $5 \% \mathrm{CO}_{2}$. HL-60 cells were treated with PBS or increasing concentrations of corilagin $(25$ and $50 \mu \mathrm{g} / \mathrm{ml})$ in a $37^{\circ} \mathrm{C}$ incubator for different durations ( $(0,1,2,3,4$ and 5 days).

Cell transfection. HL-60 cells were seeded in 6-well plates (1x10\% $/$ well) before transfection. A total of $10 \mu 1$ diluted transfection reagent DharmaFECT (Tube 1) and $100 \mathrm{nM}$ negative control (NC) inhibitor or miR-451 inhibitor (Tube 2) were mixed with $190 \mu \mathrm{l}$ serum-free DMEM for $5 \mathrm{~min}$ at room temperature. The contents of Tubes 1 and 2 were mixed and incubated for $20 \mathrm{~min}$ at room temperature, then
$1.6 \mathrm{ml}$ antibiotic-free DMEM was added for a total volume of $2 \mathrm{ml}$ transfection medium. Culture medium was replaced with transfection medium in each well. The NC inhibitor and miR-451 inhibitor were synthesized by Shanghai GenePharma Co., Ltd. Sequence of NC inhibitor was 5'-CAGUACUUUUGU GUAGUACAA-3'; sequence of miR-451 inhibitor was 5'-AAC UCAGUAAUGGUAACGGUUU-3'. Following transfection at $37^{\circ} \mathrm{C}$ for $72 \mathrm{~h}$, cells were harvested for further experiments.

Reagents and antibodies. Corilagin (purity $\geq 98 \%$ ) was purchased from Shanghai Yuanye Biotechnology Co., Ltd. DMEM, FBS and penicillin/streptomycin were purchased from Gibco (Thermo Fisher Scientific, Inc.). Cell Counting Kit-8 (CCK-8) and Carboxyfluorescein Diacetate Succinimidyl Ester (CFSE) cell proliferation kits were obtained from Dongren Chemical Technology (Shanghai) Co., Ltd.. Annexin V-APC/7-AAD apoptosis detection kit was from BioLegend, Inc., BSA, RIPA lysis buffer and $\mathrm{BCA}$ protein quantification kit were purchased from Beyotime Institute of Biotechnology. Polyvinylidene difluoride (PVDF) membrane and ECL reagent were obtained from MilliporeSigma. Primary antibodies (all 1:1,000) against light chain (LC)3 (cat. no. 12741), autophagy-related 5 (Atg5; cat. no. 12994), pro- and cleaved caspase-3 (cat. no. 14220), Bcl-xl (cat. no. 2764) and Bax (cat. no. 5023) were obtained from Cell Signalling Technology, Inc.; p62 (cat. no. 18420-1-AP, 1:1,000), Beclin-1 (cat. no. 11306-1-AP, 1:1000), $\beta$-actin (cat. no. 20536-1-AP, 1:5,000), HMGB1 (cat. no. 10829-1-AP, 1:1,000) primary and horseradish peroxidase (HRP)-conjugated secondary antibodies (both 1:5,000; goat anti-mouse IgG, cat. no. SA00001-1; goat anti-rabbit $\mathrm{IgG}$, cat. no. SA00001-2) were from ProteinTech Group, Inc. TRIzol ${ }^{\circledR}$, Alexa Fluor488-conjugated goat anti-rabbit secondary antibody (cat. no. A-11008; 1:500) and anti-fade mounting medium with DAPI were purchased from Invitrogen (Thermo Fisher Scientific, Inc.). DharmaFECT transfection reagent and RevertAid Reverse Transcription (RT) kit were obtained from Thermo Fisher Scientific, Inc. UltraSYBR Mixture was from CWBio.

Cell viability assay. HL-60 cells were seeded in 96-well plates at a density of $2 \times 10^{4}$ cells/well and cultured overnight in a $37^{\circ} \mathrm{C}$ incubator. The cells were treated with PBS or increasing concentrations of corilagin $(25$ and $50 \mu \mathrm{g} / \mathrm{ml})$ for different durations $(0,1,2,3,4$ and 5 days). CCK- 8 was added to each well and the cells were incubated for 4-6 h. The absorbance was measured at $450 \mathrm{~nm}$ using a microplate reader. Cell viability was calculated as follows: Mean optical density (OD) of treated wells-mean OD of blank wells/mean OD of control wells-mean OD of blank wells.

Flow cytometry. HL-60 cells were treated with PBS or increasing concentrations of corilagin $(25$ and $50 \mu \mathrm{g} / \mathrm{ml})$ at $37^{\circ} \mathrm{C}$ for $48 \mathrm{~h}$, collected and washed with PBS three times. For the cell proliferation assay, cells were incubated with $10 \mu \mathrm{M}$ CFSE for $10 \mathrm{~min}$ at room temperature, then heat-inactivated FBS was added to terminate the labelling reaction. The labelled cells were washed twice, resuspended in PBS and analyzed by flow cytometry (FACSCalibur; BD Biosciences). For the cell apoptosis assay, cells were stained with $5 \mu \mathrm{l}$ Annexin V-APC and 7-AAD for 
Table I. Primer sequences for reverse transcription-quantitative PCR.

\begin{tabular}{lll}
\hline Gene & \multicolumn{1}{c}{ Forward, $5^{\prime} \rightarrow 3^{\prime}$} & \multicolumn{1}{c}{ Reverse, $5^{\prime} \rightarrow 3^{\prime}$} \\
\hline miR-451 & AAACCGTTACCATTACTGAGTT & AACTCAGTAATGGTAACGGTTT \\
U6 & CTCGCTTCGGCAGCACATATACT & ACGCTTCACGAATTTGCGTGTC \\
HMGB1 & TGGTATTTTGGACTGCGGGG & TGACATTTTGCCTCTCGGCT \\
GAPDH & TGAAGGTCGGAGTCAACGG & TCCTGGAAGATGGTGATGGGA
\end{tabular}

miR, microRNA; HMGB1, high mobility group protein B1.

$15 \mathrm{~min}$ at room temperature, then assessed by flow cytometry (CytoFlex; Beckman Coulter, Inc.). The percentage of earlyand late-stage apoptotic cells was calculated using FlowJo v10 software (BD Biosciences).

Small RNA single-end sequencing. Total RNA was extracted from cells using TRIzol according to the manufacturers' instructions. The integrity, purity and concentration of RNA were detected by Agilent 2100, Nanodrop and Qubit, respectively. After the samples passed quality control tests, the Small RNA Sample Prep kit (Illumina, Inc.) was used to produce cDNA libraries. Briefly, adapters were added to the 5' and 3 ' ends of small RNA, followed by RT-quantitative (q)PCR amplification and PAGE to separate the target DNA fragments. After the library was built, the insert size was detected using Agilent 2100, and the effective concentration ( $>2 \mathrm{nM}$ ) was quantified by qPCR. The cDNA libraries were sequenced using an Illumina HiSeq 2000 platform and 50 bp single-end reads were generated. Subsequently, the raw reads were processed and filtered for the length of 21-22 nt to obtain miRNA reads. The miRNAs were aligned with miRBase (https://www.mirbase. org) and annotated using Bowtie (http://bowtie-bio.sourceforge. net). To identify the differentially expressed miRNAs, the expression levels of miRNAs were normalized using Transcripts Per Million. Normalized expression was determined as follows: Read count of miRNA x1,000,000)/read count of total miRNAs.

The criteria for identifying differentially expressed miRNAs were $\mathrm{P}<0.01$ and $\log 2$ (fold change) $>1$.

Western blotting. HL-60 cells were lysed with RIPA buffer for 30 min on ice and supernatant was collected by centrifugation at $12,000 \mathrm{x} \mathrm{g}$ for $15 \mathrm{~min}$ at $4^{\circ} \mathrm{C}$. Protein concentrations were determined using a BCA protein quantification kit. Protein (30 $\mu \mathrm{g} /$ lane) was loaded, separated via $12 \%$ SDS-PAGE (Bio-Rad Laboratories, Inc.) and transferred onto PVDF membranes. After blocking with $3 \% \mathrm{BSA}$ for $1 \mathrm{~h}$ at room temperature, the membranes were incubated with primary antibodies overnight at $4^{\circ} \mathrm{C}$ and with HRP-conjugated secondary antibodies for $1 \mathrm{~h}$ at room temperature. Finally, protein bands were exposed to ECL reagent and visualized using a chemiluminescent detector (Tanon Science and Technology Co., Ltd.). The relative protein expression was semi-quantified using Image J v.1.46r software (National Institutes of Health) using $\beta$-actin as the control.

Immunofluorescence. Cells were fixed with $4 \%$ paraformaldehyde for $10 \mathrm{~min}$ at room temperature and washed three times with PBS. After blocking with $10 \%$ FBS containing $0.1 \%$ Triton for $20 \mathrm{~min}$ at room temperature, cells were incubated with LC3 primary antibody at $4^{\circ} \mathrm{C}$ overnight and subsequently stained with 488-conjugated anti-rabbit secondary antibody at room temperature for $1 \mathrm{~h}$. Finally, cells were washed three times with PBS and mounted on glass slides using anti-fade mounting medium with DAPI. The stained cells were imaged using a confocal microscope (Zeiss LSM800; Zeiss AG; magnification, x630). The number of LC3-positive puncta/cell was quantified using ImageJ v.1.46r software (National Institutes of Health).

$R T-q P C R$. Total RNA was extracted from HL-60 cells using TRIzol and reverse transcribed to cDNA using an RT kit according to the manufacturer's protocol. RT-qPCR was performed using an UltraSYBR Mixture in a CFX-96 Touch Real-Time PCR Detection system (Bio-Rad Laboratories, Inc.). The thermocycling conditions were as follows: $95^{\circ} \mathrm{C}$ for $5 \mathrm{~min}$, followed by 40 cycles of $95^{\circ} \mathrm{C}$ for $10 \mathrm{sec}$ and $60^{\circ} \mathrm{C}$ for $30 \mathrm{sec}$. The expression level of HMGB1 was normalized to that of the reference gene $\beta$-actin, and miR-451 to that of U6. The relative expression levels of genes were calculated using the $2^{-\Delta \Delta \mathrm{Cq}}$ method (34). The primers sequences are shown in Table I.

Survival analysis of patients with AML with differentially expressed HMGBI by Gene Expression Profiling Interactive Analysis (GEPIA). The GEPIA database (http://gepia. cancer-pku.cn) was used to analyze the survival percentage of patients with AML with high or low levels of HMGB1 using 106 samples from the Genotype Tissue Expression (https://gtexportal.org) and The Cancer Genome Atlas (http://tcga-data.nci.nih.gov/tcga).

Statistical analysis. The data are presented as the mean \pm SD from three independent experiments. Data were compared using an unpaired Student's t-test or one-way ANOVA followed by Bonferroni's post hoc test using GraphPad Prism 5 (GraphPad Software, Inc.). $\mathrm{P}<0.05$ was considered to indicate a statistically significant difference.

\section{Results}

Corilagin inhibits proliferation of HL-60 AML cells. The inhibitory effect of corilagin on AML cells was analyzed using CCK-8 and CFSE assay kits. The AML cell line HL-60 was treated with PBS or increasing concentrations of 
A

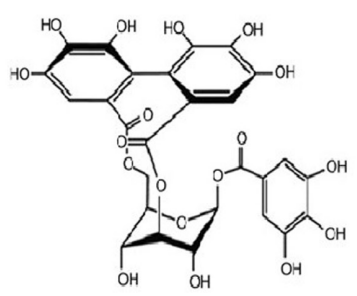

C

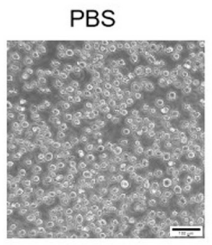

$25 \mu \mathrm{g} / \mathrm{ml}$ corilagin

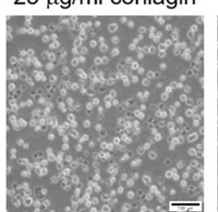

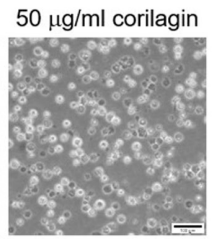

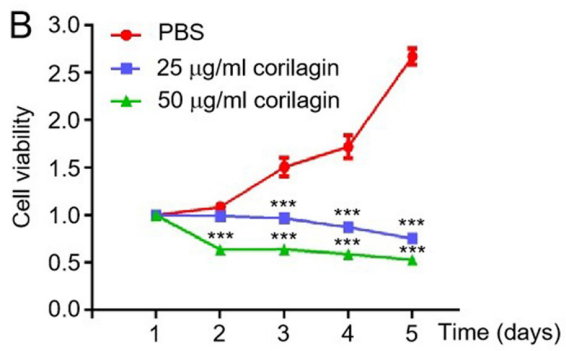

D

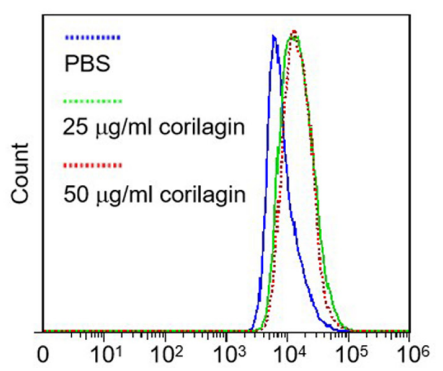

Figure 1. Corilagin inhibits proliferation of HL-60 cells. (A) Chemical structure of corilagin. (B) HL-60 cells were treated with increasing concentrations of corilagin for different durations. Cell viability was analyzed by Cell Counting Kit- 8 assay. ${ }^{* * *}$ P $<0.001$ vs. PBS group. (C) Optical microscopy observation of HL-60 cells following $48 \mathrm{~h}$ treatment with PBS or corilagin. Scale bar, $100 \mu \mathrm{m}$. (D) Flow cytometry analysis of HL-60 cells using carboxyfluorescein diacetate succinimidyl ester following $48 \mathrm{~h}$ treatment with PBS or corilagin.

corilagin (25 and $50 \mu \mathrm{g} / \mathrm{ml}$ ) for different durations (Fig. 1A). Corilagin significantly inhibited proliferation of HL-60 cells in a dose- and time-dependent manner (Fig. 1B). The cytotoxicity of corilagin on HL-60 cells was also evaluated by microscopy. The cell number was notably decreased and the cell shape became irregular following corilagin treatment for $48 \mathrm{~h}$ (Fig. 1C). Moreover, the fluorescence intensity of the corilagin-treated group was higher than that of the PBS group (Fig. 1D). These data indicated that corilagin inhibited the proliferation of HL-60 AML cells.

Corilagin activates apoptosis in HL-60 AML cells. To investigate the apoptotic effect of corilagin on AML cells, Annexin V/7-AAD staining assay was used to assess apoptotic cell death by flow cytometry. HL-60 cells were treated with PBS or different concentrations of corilagin $(25$ and $50 \mu \mathrm{g} / \mathrm{ml})$ for $48 \mathrm{~h}$. The percentage of early- and late-stage apoptotic cells increased following corilagin treatment in a dose-dependent manner (Fig. 2A and B). To verify whether corilagin-induced apoptosis was triggered by the intrinsic signalling pathway, the expression levels of key proteins were detected by western blotting. Corilagin increased the protein levels of cleaved caspase- 3 and Bak and decreased the level of Bcl-xl, indicating that the intrinsic mitochondrial apoptosis pathway was activated following exposure to corilagin (Fig. 2C and D).

Corilagin inhibits autophagy of HL-60 AML cells. Autophagy may function as a protective mechanism in tumor cells, and inhibition of autophagy promotes cell apoptosis (30). Microtubule-associated protein LC3 is a key autophagy-associated protein involved in autophagosome formation. Cytosolic LC3-I is converted to membrane-bound LC3-II by conjugation to phosphatidylethanolamine and localizes to the autophagosome (35). To determine the effect of corilagin on AML cell autophagy, the ratio of LC3-II/I and number of LC3-positive puncta were assessed by western blotting and immunofluorescence, respectively. Corilagin significantly decreased the conversion of LC3-I into LC3-II and formation of LC3-positive puncta. In addition, the expression levels of Atg5 and Beclin-1 decreased, while p62 expression increased following corilagin treatment. These results suggested that corilagin exhibited an inhibitory effect on AML cell autophagy (Fig. 3A-D).

Corilagin elevates expression of tumor suppressor miR-451. Previous studies have revealed that corilagin prevents liver fibrosis by blocking the miR-21-regulated Smad signalling pathway $(36,37)$. To assess changes in miRNA expression levels caused by corilagin, small RNA sequencing was performed. Compared with the PBS group, eight miRNAs were notably upregulated and two were downregulated in the corilagin-treated groups. In addition, the expression levels of the differentially expressed miRNAs showed a dose-dependent change when treated with corilagin (Fig. 4A). miR-451 has been reported to be abnormally downregulated in patients with AML and serves as a tumor suppressor by inducing apoptosis $(25,26)$. The present microRNA sequencing data showed that miR-451 was notably upregulated following exposure to corilagin. RT-qPCR results verified the elevated expression of miR-451 following corilagin treatment in a dose-dependent manner (Fig. 4B).

Corilagin downregulates HMGB1 expression by increasing $m i R-451$. To determine the mechanisms by which miR-451 regulates AML, target genes of miR-451 were screened. A previous study reported that the expression of HMGB1 was negatively regulated by miR-451 during cardiomyocyte A/R injury (27). The GEPIA database showed that HMGB1 levels were negatively associated with survival of patients with AML. The low HMGB1 expression group had a higher survival rate than the high HMGB1 expression 
A

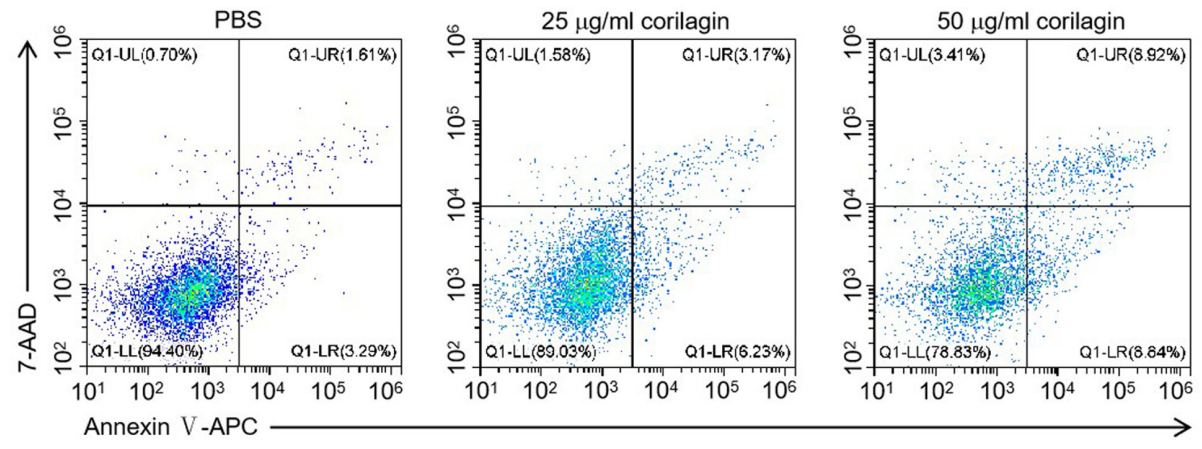

B

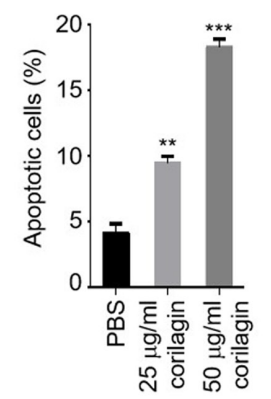

C

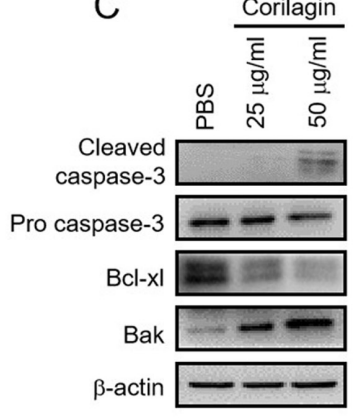

D $\quad$ PBS

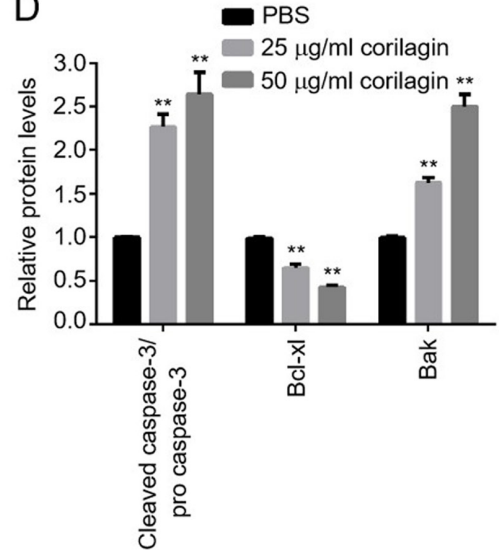

Figure 2. Corilagin activates apoptosis in HL-60 cells. (A) Flow cytometry analysis of apoptosis in HL-60 cells following treatment with PBS or 25 and $50 \mu \mathrm{g} / \mathrm{ml}$ corilagin for $48 \mathrm{~h}$. (B) Proportion of apoptotic cells. (C) Expression levels of the apoptosis-associated proteins cleaved caspase-3, pro caspase-3, Bcl-xl and Bak in corilagin-treated HL-60 cells were analyzed by western blotting. (D) Relative protein expression of cleaved caspase-3/pro caspase-3, Bcl-xl and Bak were calculated using Image $\mathrm{J} .{ }^{* *} \mathrm{P}<0.01,{ }^{* * *} \mathrm{P}<0.001$ vs. PBS group.

group (Fig. 5A). To test whether miR-451 functions by regulating HMGB1 during corilagin treatment, the expression of HMGB1 was measured using RT-qPCR and western blotting. The mRNA and protein levels of HMGB1 were significantly decreased in response to corilagin in a dose-dependent manner (Fig. 5B-D). Knockdown of miR-451 decreased the corilagin-induced downregulation of HMGB1, indicating a negative regulation of HMGB1 by miR-451 during corilagin treatment (Fig. 5E and F).

Corilagin inhibits proliferation of HL-60 cells via the miR-451/HMGB1 axis. To investigate whether regulation of miR-451/HMGB1 axis by corilagin is involved in inhibition of HL-60 cell proliferation, miR-451 was knocked down and cell proliferation was analyzed. The CCK-8 assay showed that knockdown of miR-451 promoted proliferation of HL-60 cells, indicating a tumor suppressive effect of miR-451 on AML cells. Compared with corilagin treatment-alone, knockdown of miR-451 attenuated corilagin-induced inhibition of HL-60 cell proliferation, which implied that miR-451 was involved in corilagin-induced inhibition of proliferation (Fig. 6A and B). A recent study showed that miR-451 enhances death of AML cells by targeting HMGB1 (38). As aforementioned, the present results also suggested negative regulation of HMGB1 by miR-451 during corilagin treatment (Fig. 5E and F). Therefore, it was concluded that corilagin inhibited the proliferation of HL-60 cells via the miR-451/HMGB1 axis.

\section{Discussion}

Corilagin, a water-soluble tannin, is the primary bioactive ingredient of the E. phyllanthus plant. Corilagin possesses anti-viral, anti-inflammatory, antioxidant and antitumor properties (10-12). Corilagin inhibits proliferation of a variety of cancer cells, including hepatic carcinoma, as well as breast, gastric and ovarian cancer (15-17). To the best of our knowledge, however, the effect of corilagin on leukemia cells has rarely been reported. In the present study, corilagin significantly inhibited proliferation of HL-60 AML cells in a concentration- and time-dependent manner.

Apoptosis, a type of programmed cell death, is a key mechanism underlying the activity of numerous antitumor drugs, such as oblimersen sodium and nutlins (39-41). The present results indicated that the suppressive effect of corilagin on HL-60 cells was associated with apoptotic cell death. Flow cytometry analysis of Annexin V/7-AAD staining showed that the percentage of early- and late-stage apoptotic cells increased following corilagin treatment in a dose-dependent manner. Apoptosis is primarily regulated by the mitochondria-mediated intrinsic, death receptor-mediated extrinsic and endoplasmic reticulum stress-induced apoptotic pathways (42). The intrinsic pathway is accompanied by activation of pro-apoptotic proteins (Bax and Bak), inhibition of anti-apoptotic proteins (Bcl-2 and Bcl-xl) and cleavage of procaspases-3/6/7, leading to cell death. In the present study, corilagin increased protein levels of cleaved caspase- 3 and Bak and decreased levels of Bcl-xl, 
A

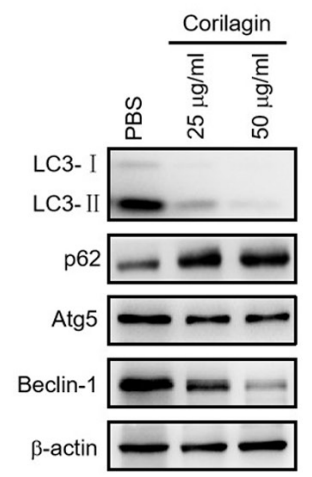

C
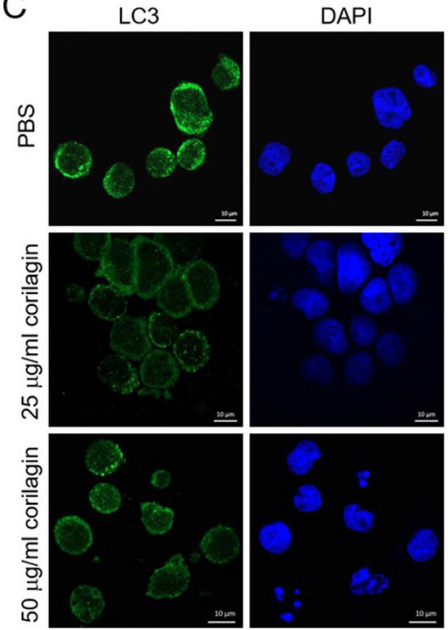

Merge
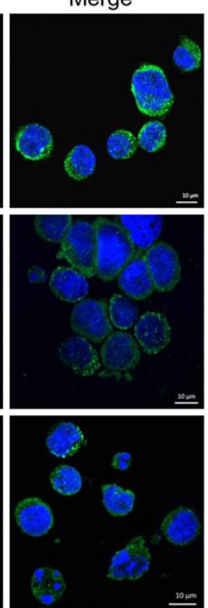

B

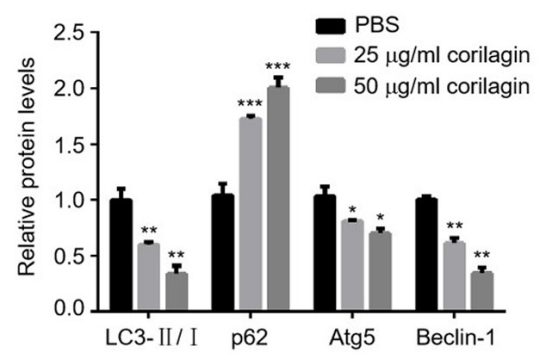

D

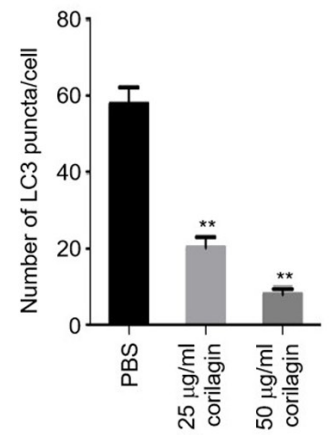

Figure 3. Corilagin inhibits autophagy of HL-60 cells. (A) HL-60 cells were incubated with PBS or 25 and $50 \mu \mathrm{g} / \mathrm{ml}$ corilagin for $48 \mathrm{~h}$ and expression of autophagy-associated proteins LC3, p62, Atg5 and Beclin-1 was determined by western blotting. (B) Relative protein expression levels of LC3-II/LC3-I, p62, Atg5 and Beclin-1 were quantified using Image J software. (C) Confocal images of LC3-positive puncta, representing autophagosomes. Scale bar, $10 \mu \mathrm{m}$. (D) Quantification of the average number of LC3-positive puncta per cell using Image J software. ${ }^{*} \mathrm{P}<0.05,{ }^{* *} \mathrm{P}<0.01,{ }^{* * * *} \mathrm{P}<0.001$ vs. PBS group. LC, light chain; Atg, autophagy-related.
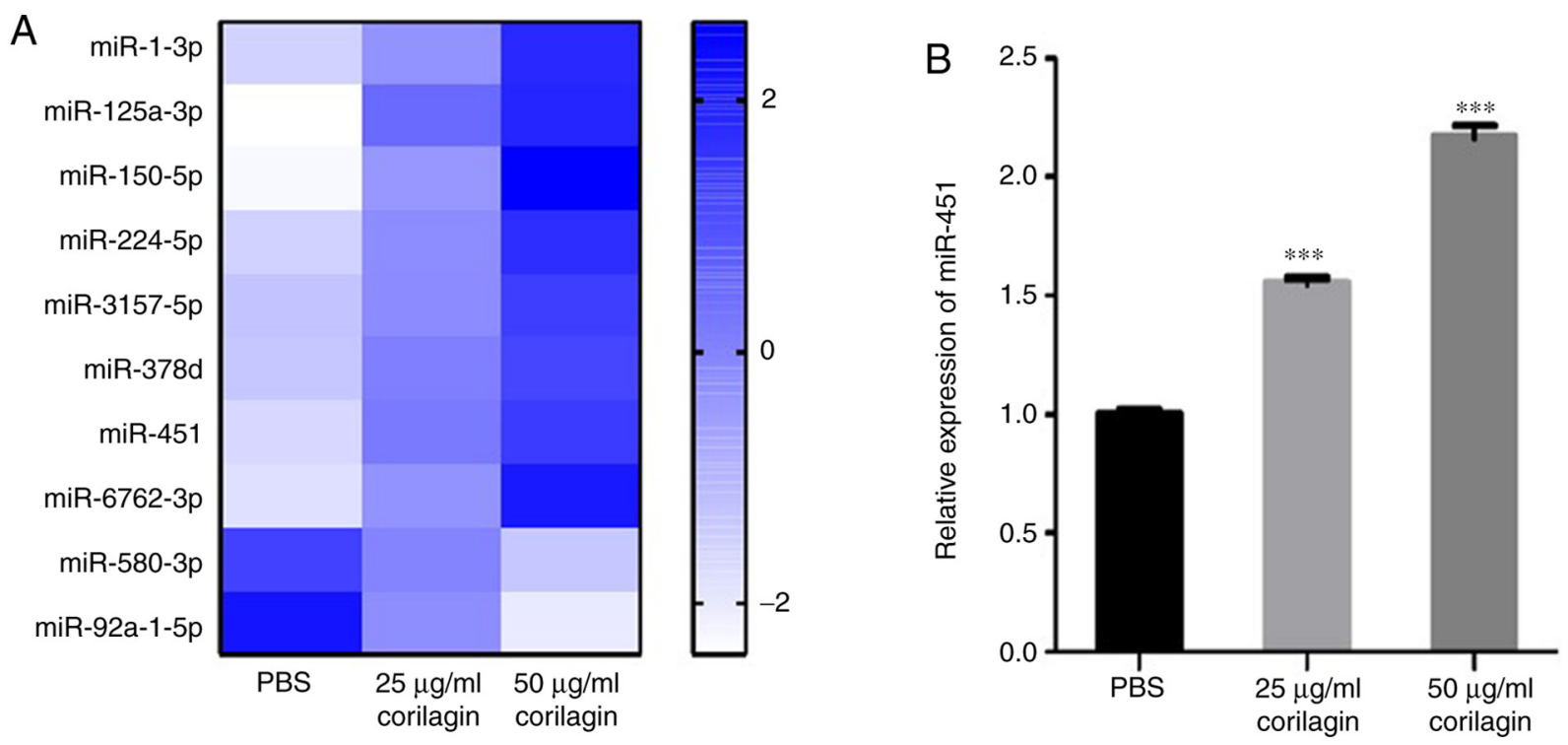

Figure 4. Corilagin elevates expression of tumor suppressor miR-451. (A) Heatmap analysis of miR expression profiles in HL-60 cells treated with PBS or 25 and $50 \mu \mathrm{g} / \mathrm{ml}$ corilagin for $48 \mathrm{~h}$. (B) Expression of miR-451 was validated by reverse transcription-quantitative PCR in HL-60 cells treated with PBS or 25 and $50 \mu \mathrm{g} / \mathrm{ml}$ corilagin for $48 \mathrm{~h} .{ }^{* * *} \mathrm{P}<0.001$ vs. PBS group. miR, microRNA.

indicating that the intrinsic mitochondrial apoptosis pathway was activated following exposure to corilagin.
Autophagy is a highly conserved process that maintains homeostasis by degrading and recycling protein aggregates, 


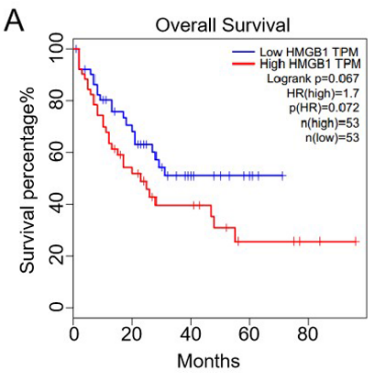

B

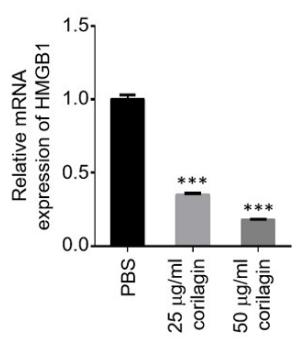

C

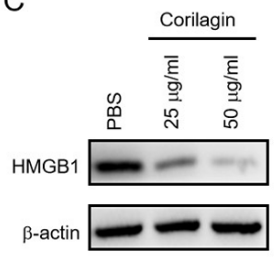

D

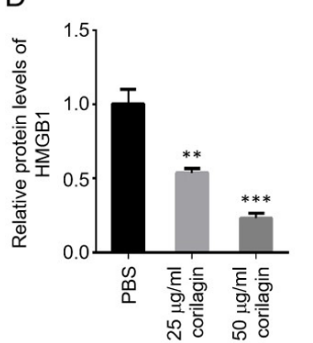

E

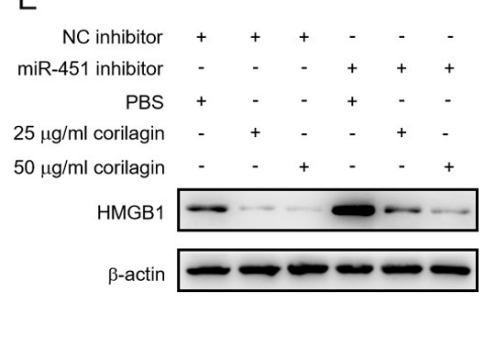

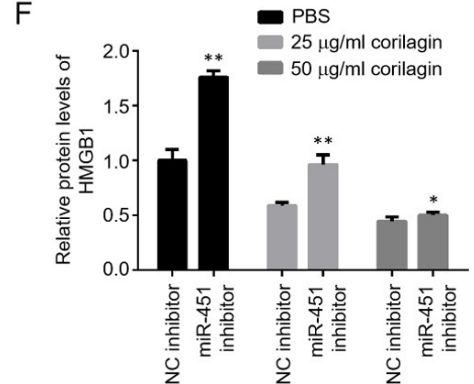

Figure 5. Corilagin downregulates HMGB1 expression by increasing miR-451. (A) Comparison of survival percentage between low- and high-HMGB1 expression groups by GEPIA database. HL-60 cells were treated with PBS or 25 and $50 \mu \mathrm{g} / \mathrm{ml}$ corilagin for $48 \mathrm{~h}$. The (B) mRNA and (C) protein level of HMGB1 were detected by reverse transcription-quantitative PCR and western blotting, respectively. (D) Relative protein expression of HMGB1 was calculated using Image $\mathrm{J}$ software. ${ }^{* * *} \mathrm{P}<0.01,{ }^{* * * *} \mathrm{P}<0.001$ vs. PBS group. HL-60 cells were transfected with NC inhibitor or miR-451 inhibitor, following by treatment with corilagin for $48 \mathrm{~h}$. The protein expression of HMGB1 was analyzed by (E) western blotting and (F) Image J software. ${ }^{*} \mathrm{P}<0.05,{ }^{* *} \mathrm{P}<0.01 \mathrm{vs}$. NC inhibitor. HMGB1, high mobility group protein B1; NC, negative control.
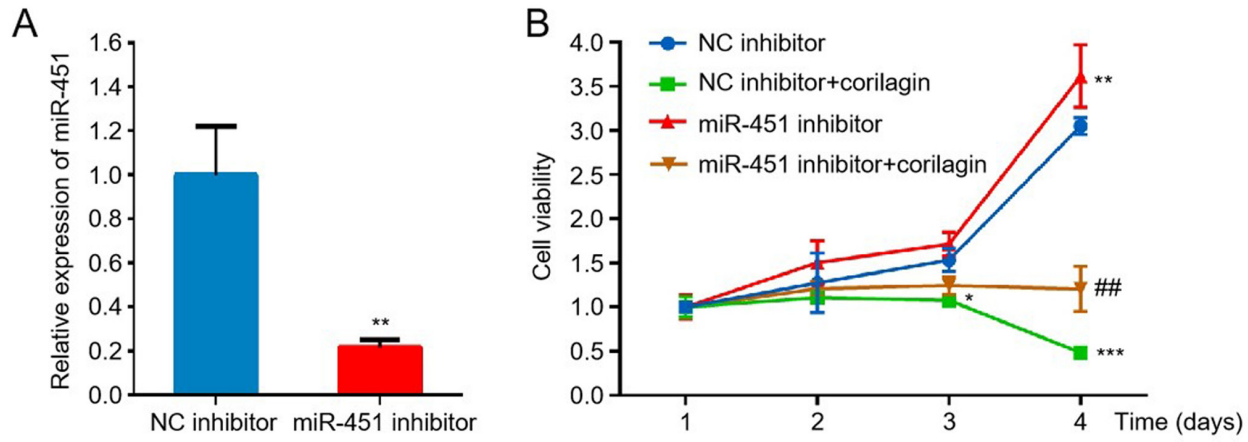

Figure 6. Corilagin inhibits proliferation of HL-60 cells via the miR-451/high mobility group protein B1 axis. HL-60 cells were transfected with NC or miR-451 inhibitor. (A) Transfection efficiency was detected by reverse transcription-quantitative PCR. (B) Following treatment with $25 \mu \mathrm{g} / \mathrm{ml}$ corilagin, cell viability was analyzed by Cell Counting Kit- 8 assay. ${ }^{*} \mathrm{P}<0.05,{ }^{* *} \mathrm{P}<0.01,{ }^{* * *} \mathrm{P}<0.001$ vs. NC inhibitor, ${ }^{* \#} \mathrm{P}<0.01$ vs. NC inhibitor + corilagin. NC, negative control; miR, microRNA.

long-lived proteins and damaged organelles (43). Under stress, such as nutrient deprivation, hypoxia, chemotherapy and radiotherapy, autophagy is a survival mechanism that provides nutrients for vital cellular functions and protects cells from apoptosis or necrosis (43). There is crosstalk between autophagy and apoptosis and inhibition of autophagy may lead to apoptotic cell death $(44,45)$. The present results showed that corilagin markedly decreased the conversion of LC3-I into LC3-II and the formation of LC3 puncta, leading to the accumulation of substrate $\mathrm{p} 62$. These data suggested that corilagin exhibited an inhibitory effect on AML cell autophagy; further research should investigate the association between autophagy and corilagin-induced apoptosis.

Numerous types of miRNA are aberrantly expressed in AML, including miR-181, miR-21, miR-34 and miR-451 (20). miR-451, which serves as a tumor suppressor by suppressing proliferation of malignant cells and inducing apoptosis, is abnormally downregulated in patients with AML. Previous studies have showed that miR-451 inhibits AML development by targeting tyrosine 3-monooxygenase/tryptophan 5-monooxygenase activation protein $\zeta$ and heterogeneous nuclear ribonucleoprotein A1 $(25,26)$. However, a recent study found that miR-451 enhances the death of AML cells by targeting HMGB1 (38). HMGB1 levels in the serum of patients with AML increase significantly following chemotherapy, and inhibition of HMGB1 improves the sensitivity of AML cells to chemotherapy drugs $(24,33)$.

The present study investigated whether corilagin exerts its anti-proliferative effects by regulating the miR-451/HMGB1 axis. microRNA sequencing and RT-qPCR both demonstrated that corilagin notably elevated the expression of miR-451 in a concentration-dependent 
manner, while mRNA and protein levels of HMGB1 were markedly decreased in response to corilagin in a dose-dependent manner. Knockdown of miR-451 decreased corilagin-induced downregulation of HMGB1, indicating negative regulation of HMGB1 by miR-451 following corilagin treatment. Furthermore, knockdown of miR-451 also attenuated corilagin-induced inhibition of HL-60 cell proliferation, implying that miR-451 was involved in the inhibitory effect of corilagin on cell proliferation. Therefore, it was hypothesized that corilagin may function in AML treatment by regulating the miR-451/HMGB1 axis.

In conclusion, the present study demonstrated the inhibitory effects of corilagin on HL-60 AML cell proliferation and viability in a dose- and time-dependent manner. Corilagin induced apoptosis in HL-60 cells by activating the intrinsic mitochondrial apoptosis pathway. Moreover, inhibition of autophagy in HL-60 cells induced by corilagin may be associated with apoptotic cell death. Corilagin modulated miR-451 and HMGB1 expression, and knockdown of miR-451 alleviated corilagin-induced downregulation of HMGB1 and inhibition of HL-60 cell proliferation, indicating that corilagin may exert its anticancer effects by regulating the miR-451/HMGB1 axis. These results suggested that corilagin may be a potential novel drug for treating AML.

\section{Acknowledgements}

Not applicable.

\section{Funding}

The present study was supported by the Science and Technology Development Program of Medical and Health from Shandong Province (grant no. 2018WSB34003), PhD Research Foundation of Affiliated Hospital of Jining Medical University (grant no. 2018-BS-011) and Incubation Project of National Natural Science Foundation of China (grant no. 700103005$)$.

\section{Availability of data and materials}

The datasets used and/or analyzed during the current study are available from the authors on reasonable request.

\section{Authors' contributions}

SJ and DH performed experiments and interpreted data. XL, $\mathrm{PC}, \mathrm{JL}, \mathrm{MC}$ and $\mathrm{CW}$ performed the experiments. SJ conceived the study, directed the experiments and wrote the manuscript. $\mathrm{CM}$ and $\mathrm{HZ}$ designed and conceived the study. SJ and DH confirm the authenticity of all the raw data. All authors have read and approved the final manuscript.

\section{Ethics approval and consent to participate}

Not applicable.

\section{Patient consent for publication}

Not applicable.

\section{Competing interests}

The authors declare that they have no competing interests.

\section{References}

1. Dohner H, Weisdorf DJ and Bloomfield CD: Acute myeloid leukemia. N Engl J Med 373: 1136-1152, 2015.

2. Khwaja A, Bjorkholm M, Gale RE, Levine RL, Jordan CT, Ehninger G, Bloomfield CD, Estey E, Burnett A, Cornelissen JJ, et al: Acute myeloid leukaemia. Nat Rev Dis Primers 2: 16010, 2016.

3. Sasine JP and Schiller GJ: Emerging strategies for high-risk and relapsed/refractory acute myeloid leukemia: Novel agents and approaches currently in clinical trials. Blood Rev 29: 1-9, 2015.

4. Shafer D and Grant S: Update on rational targeted therapy in AML. Blood Rev 30: 275-283, 2016.

5. Nair R, Salinas-Illarena A and Baldauf HM: New strategies to treat AML: Novel insights into AML survival pathways and combination therapies. Leukemia 35: 299-311, 2021.

6. Short NJ, Konopleva M, Kadia TM, Borthakur G, Ravandi F, DiNardo CD and Daver N: Advances in the treatment of acute myeloid leukemia: New drugs and new challenges. Cancer Discov 10: 506-525, 2020.

7. Dohner H, Estey EH, Amadori S, Appelbaum FR, Buchner T, Burnett AK, Dombret H, Fenaux P, Grimwade D, Larson RA, et al: Diagnosis and management of acute myeloid leukemia in adults: Recommendations from an international expert panel, on behalf of the European LeukemiaNet. Blood 115: 453-474, 2010.

8. Karathedath S, Rajamani BM, Musheer Aalam SM, Abraham A, Varatharajan S, Krishnamurthy P, Mathews V, Velayudhan SR and Balasubramanian P: Role of NF-E2 related factor 2 (Nrf2) on chemotherapy resistance in acute myeloid leukemia (AML) and the effect of pharmacological inhibition of Nrf2. PLoS One 12: e0177227, 2017.

9. Shafat MS, Gnaneswaran B, Bowles KM and Rushworth SA: The bone marrow microenvironment-Home of the leukemic blasts. Blood Rev 31: 277-286, 2017.

10. Tong F, Zhang J, Liu L, Gao X, Cai Q, Wei C, Dong J, Hu Y, Wu G and Dong X: Corilagin attenuates radiation-induced brain injury in mice. Mol Neurobiol 53: 6982-6996, 2016.

11. Li X, Deng Y, Zheng Z, Huang W, Chen L, Tong Q and Ming Y: Corilagin, a promising medicinal herbal agent. Biomed Pharmacother 99: 43-50, 2018.

12. Lu J, Ye C, Huang Y, Huang D, Tang L, Hou W, Kuang Z, Chen Y, Xiao S, Yishake M and He R: Corilagin suppresses RANKL-induced osteoclastogenesis and inhibits oestrogen deficiency-induced bone loss via the NF- $\kappa \mathrm{B}$ and PI3K/AKT signalling pathways. J Cell Mol Med 24: 10444-10457, 2020.

13. Gambari R, Borgatti M, Lampronti I, Fabbri E, Brognara E, Bianchi N, Piccagli L, Yuen MC, Kan CW, Hau DK, et al: Corilagin is a potent inhibitor of NF-kappaB activity and downregulates TNF-alpha induced expression of IL-8 gene in cystic fibrosis IB3-1 cells. Int Immunopharmacol 13: 308-315, 2012.

14. Xiao HT, Lin CY, Ho DH, Peng J, Chen Y, Tsang SW, Wong M, Zhang XJ, Zhang $M$ and Bian ZX: Inhibitory effect of the gallotannin corilagin on dextran sulfate sodium-induced murine ulcerative colitis. J Nat Prod 76: 2120-2125, 2013.

15. MilaniR,BrognaraE, FabbriE, Finotti A,Borgatti M,Lampronti I, Marzaro G, Chilin A, Lee KK, Kok SH, et al: Corilagin induces high levels of apoptosis in the temozolomide-resistant T98G glioma cell line. Oncol Res 26: 1307-1315, 2018.

16. Tong Y, Zhang G, Li Y, Xu J, Yuan J, Zhang B, Hu T and Song G: Corilagin inhibits breast cancer growth via reactive oxygen species-dependent apoptosis and autophagy. J Cell Mol Med 22: 3795-3807, 2018.

17. Jia L, Jin H, Zhou J, Chen L, Lu Y, Ming Y and Yu Y: A potential anti-tumor herbal medicine, Corilagin, inhibits ovarian cancer cell growth through blocking the TGF- $\beta$ signaling pathways. BMC Complement Altern Med 13: 33, 2013.

18. Emmrich S, Katsman-Kuipers JE, Henke K, Khatib ME, Jammal R, Engeland F, Dasci F, Zwaan CM, den Boer ML, Verboon L, et al: MiR-9 is a tumor suppressor in pediatric AML with $\mathrm{t}(8 ; 21)$. Leukemia 28: 1022-1032, 2014.

19. So AY, Sookram R, Chaudhuri AA, Minisandram A, Cheng D, Xie C, Lim EL, Flores YG, Jiang S, Kim JT, et al: Dual mechanisms by which miR-125b represses IRF4 to induce myeloid and B-cell leukemias. Blood 124: 1502-1512, 2014. 
20. Liu Y, Cheng Z, Pang Y, Cui L, Qian T, Quan L, Zhao H, Shi J, Ke X and Fu L: Role of microRNAs, circRNAs and long noncoding RNAs in acute myeloid leukemia. J Hematol Oncol 12: 51, 2019.

21. Jiang X, Hu C, Arnovitz S, Bugno J, Yu M, Zuo Z, Chen P, Huang H, Ulrich B, Gurbuxani S, et al: MiR-22 has a potent anti-tumour role with therapeutic potential in acute myeloid leukaemia. Nat Commun 7: 11452, 2016.

22. Wallace JA and O'Connell RM: MicroRNAs and acute myeloid leukemia: Therapeutic implications and emerging concepts. Blood 130: 1290-1301, 2017.

23. Liu L, Ren W and Chen K: MiR-34a promotes apoptosis and inhibits autophagy by targeting HMGB1 in acute myeloid leukemia cells. Cell Physiol Biochem 41: 1981-1992, 2017.

24. Zhang Y, Liu Y and Xu X: Upregulation of miR-142-3p improves drug sensitivity of acute myelogenous leukemia through reducing P-glycoprotein and repressing autophagy by targeting HMGB1. Transl Oncol 10: 410-418, 2017.

25. Su R, Gong JN, Chen MT, Song L, Shen C, Zhang XH, Yin XL, Ning HM, Liu B, Wang F, et al: c-Myc suppresses miR-451 dash, verticalYWTAZ/AKT axis via recruiting HDAC3 in acute myeloid leukemia. Oncotarget 7: 77430-77443, 2016.

26. Song L, Lin HS, Gong JN, Han H, Wang XS, Su R, Chen MT, Shen C, Ma YN, Yu J and Zhang JW: MicroRNA-451-modulated hnRNP A1 takes a part in granulocytic differentiation regulation and acute myeloid leukemia. Oncotarget 8: 55453-55466, 2017.

27. Cao J, Da Y, Li H, Peng Y and Hu X: Upregulation of microRNA-451 attenuates myocardial I/R injury by suppressing HMGB1. PLoS One 15: e0235614, 2020.

28. Amato J, Cerofolini L, Brancaccio D, Giuntini S, Iaccarino N, Zizza P, Iachettini S, Biroccio A, Novellino E, Rosato A, et al: Insights into telomeric G-quadruplex DNA recognition by HMGB1 protein. Nucleic Acids Res 47: 9950-9966, 2019.

29. Kang R, Chen R, Zhang Q, Hou W, Wu S, Cao L, Huang J, Yu Y, Fan XG, Yan Z, et al: HMGB1 in health and disease. Mol Aspects Med 40: 1-116, 2014.

30. Kang R, Livesey KM, Zeh HJ, Loze MT and Tang D: HMGB1: A novel Beclin 1-binding protein active in autophagy. Autophagy 6: 1209-1211, 2010

31. Pan B, Chen D, Huang J, Wang R, Feng B, Song H and Chen L: HMGB1-mediated autophagy promotes docetaxel resistance in human lung adenocarcinoma. Mol Cancer 13: 165, 2014.

32. Hou X, Yang C, Zhang L, Hu T, Sun D, Cao H, Yang F, Guo G, Gong C, Zhang X, et al: Killing colon cancer cells through PCD pathways by a novel hyaluronic acid-modified shell-core nanoparticle loaded with RIP3 in combination with chloroquine. Biomaterials 124: 195-210, 2017.

33. Liu L, Zhang J, Zhang X, Cheng P, Liu L, Huang Q, Liu H, Ren S, Wei P, Wang C, et al: HMGB1: An important regulator of myeloid differentiation and acute myeloid leukemia as well as a promising therapeutic target. J Mol Med (Berl) 99: 107-118, 2021
34. Livak KJ and Schmittgen TD: Analysis of relative gene expression data using real-time quantitative PCR and the 2(-Delta Delta C(T)) Method. Methods 25: 402-408, 2001.

35. Huang R, Xu Y, Wan W, Shou X, Qian J, You Z, Liu B, Chang C, Zhou T, Lippincott-Schwartz J and Liu W: Deacetylation of nuclear LC3 drives autophagy initiation under starvation. Mol Cell 57: 456-466, 2015.

36. Yang F, Wang Y, Xue J, Ma Q, Zhang J, Chen YF, Shang ZZ,Li QQ, Zhang SL andZhaoL:Effect of corilagin on the miR-21/smad7/ERK signaling pathway in a schistosomiasis-induced hepatic fibrosis mouse model. Parasitol Int 65: 308-315, 2016.

37. Zhou X, Xiong J, Lu S, Luo L, Chen ZL, Yang F, Jin F, Wang Y, Ma Q, Luo YY, et al: Inhibitory effect of corilagin on miR-21-regulated hepatic fibrosis signaling pathway. Am J Chin Med 47: 1541-1569, 2019.

38. Zhang Y, Chu X and Wei Q: MiR-451 promotes cell apoptosis and inhibits autophagy in pediatric acute myeloid leukemia by targeting HMGB1. J Environ Pathol Toxicol Oncol 40: 45-53, 2021.

39. Pistritto G, Trisciuoglio D, Ceci C, Garufi A and D'Orazi G: Apoptosis as anticancer mechanism: Function and dysfunction of its modulators and targeted therapeutic strategies. Aging (Albany NY) 8: 603-619, 2016.

40. Rai KR, Moore J, Wu J, Novick SC and O'Brien SM: Effect of the addition of oblimersen (Bcl-2 antisense) to fludarabine/cyclophosphamide for replased/refractory chronic lymphocytic leukaemia (CLL) on survival in patients who achieve CR/nPR: Five-year follow-up from a randomized phase III study. J Clin Oncol 26 (Suppl 15): S7008, 2008.

41. Shangary S and Wang S: Small-molecule inhibitors of the MDM2-p53 protein-protein interaction to reactivate $\mathrm{p} 53$ function: A novel approach for cancer therapy. Annu Rev Pharmacol Toxicol 49: 223-241, 2008.

42. Hu H, Tian M, Ding C and Yu S: The C/EBP homologous protein (CHOP) transcription factor functions in endoplasmic reticulum stress-induced apoptosis and microbial infection. Front Immunol 9: 3083, 2018.

43. Dikic I and Elazar Z: Mechanism and medical implications of mammalian autophagy. Nat Rev Mol Cell Biol 19: 349-364, 2018.

44. Maiuri MC, Zalckvar E, Kimchi A and Kroemer G: Self-eating and self-killing: Crosstalk between autophagy and apoptosis. Nat Rev Mol Cell Biol 8: 741-752, 2007.

45. Li Q, Yin Y, Zheng Y, Chen F and Jin P: Inhibition of autophagy promoted high glucose/ROS-mediated apoptosis in ADSCs. Stem Cell Res Ther 9: 289, 2018.

(i) $\Theta$ This work is licensed under a Creative Commons Attribution-NonCommercial-NoDerivatives 4.0 International (CC BY-NC-ND 4.0) License. 